Scientific Paper

\title{
Effective energy measurement using radiochromic film: application of a mobile scanner
}

\author{
Tatsuhiro GotANDA ${ }^{1, a}$, Toshizo KATSUdA ${ }^{2}$, Rumi GOTANDA ${ }^{3}$, Tadao KUWANO ${ }^{4}$, Takuya AKAGAWA ${ }^{5}$, Nobuyoshi \\ TANKI ${ }^{2}$, Akihiko TABUCHI 6 , Tetsunori SHIMONO ${ }^{1}$, Yasuyuki KAWAJI', Yoshihiro TAKEDA ${ }^{4}$ \\ ${ }^{I}$ Department of Radiological Science, Faculty of Health Sciences, Junshin Gakuen University, Fukuoka, Japan \\ ${ }^{2}$ Department of Radiological Technology, Faculty of Health Sciences, Butsuryo College of Osaka, Osaka, Japan \\ ${ }^{3}$ Department of Radiological Sciences, Ibaraki Prefectural University of Health Sciences, Ibaraki, Japan \\ ${ }^{4}$ Department of Radiological Technology, Graduate School of Health Sciences, Okayama University, Okayama, Japan \\ ${ }^{5}$ Department of Radiological Technology, Tokushima Red Cross Hospital, Tokushima, Japan \\ ${ }^{6}$ Department of Radiology, Kawasaki Medical School Kawasaki Hospital, Okayama, Japan \\ ${ }^{a}$ E-mail address: gotanda.t@junshin-u.ac.jp
}

(received 19 July 2016; revised 27 October 2016; accepted 3 November 2016)

\begin{abstract}
The effective energy calculated using the half-value layer (HVL) is an important parameter for quality assurance (QA) and quality control (QC). However constant monitoring has not been performed because measurements using an ionization chamber (IC) are time-consuming and complicated. To solve these problems, a method using radiochromic film (GAFCHROMIC EBT2 dosimetry film (GAF-EBT2) with slight energy dependency errors), a mobile scanner and step-shaped aluminum (SSAl) filter is developed. The results of the method using a mobile scanner were compared with those of the recommended method using an IC in order to evaluate its applicability. The difference ratios of the effective energies by each method using a mobile scanner with GAF-EBT2 were less than 5\% compared with results of an IC. It is considered that this method offers a simple means of determining HVL for QA and QC consistently and quickly without the need for an IC dosimeter.
\end{abstract}

Key words: radiochromic film; effective energy; half-value layer; mobile scanner; flat bed scanner.

\section{Introduction}

The effective energy of X-ray beams is one of a number of standard quality assurance (QA) and quality control (QC) tests for various $\mathrm{X}$-ray imaging systems [1]. In the radiation quality management of complex X-ray generators, the half-value layer (HVL), which is used to calculate the effective energy, is important. In general, HVLs are measured by means of an ionization chamber (IC) dosimeter, and this is referred to as the standard method. However, the HVL is not monitored constantly. The main reason for this is the time-consuming nature of the process owing to the number of measurement points. In addition, not all institutions have an IC dosimeter.

Radiochromic films can easily be used to measure absorbed doses, because they do not require development processing and indicate a density change that depends on the absorbed dose [2,3]. Therefore, they are used in HVL measurement techniques [4-6]. One of the main characteristics of radiochromic film that sets it apart from the IC dosimeter is that it can be used to measure two-dimensional dose distributions, as well as serving as a general X-ray film.
Another principal characteristic is the calibration curve of absorbed dose versus film density. The calibration curve takes various forms depending on the type of radiochromic films [7,8]. The calibration curve of GAFCHROMIC EBT2 (GAFEBT2: International Specialty Products, Wayne, NJ) shows minimal curvature in the recommended dose range by manufacture [7]. Therefore, it is logical to consider that the calibration curve would be a straight line in the low range for HVL measurement. It is not necessary for film density to be converted into an absorbed dose, because the calibration curve is a straight line in the low dose range for HVL measurements of X-rays in the diagnostic range [6]. Therefore, the HVL can be evaluated using only the ratio of the density distribution in the radiochromic film [4]. On the basis of these characteristics, the HVL could be evaluated easily and quickly using the density distribution and a step-shaped $\mathrm{Al}$ (SSAl) filter, in what we refer to as the method using GAFCHROMIC XR TYPE-R (GAF-R: International Specialty Products, Wayne, NJ) [5,6].

However, there some problems that need to be solved for each measurement method using GAF-R. The attenuation curve using GAF-R, as one type of radiochromic film, shows a 
lower value at the thicker end of the $\mathrm{Al}$ filter as compared to that obtained by the standard method using an IC dosimeter. The energy-response characteristic of GAF-R is minimal over the $75-150 \mathrm{kVp}$ range. However, the sensitivity of GAF-R is highest at approximately $50 \mathrm{keV}$, decreasing at higher energies $[9,10]$. Higher energies were provided by the thicker steps of the $\mathrm{Al}$ filter, and it is considered that this is responsible for the error of lower values at the thicker end of the Al filter. Therefore, measurement of the second half-value layer was impossible due to the energy-dependent influence in the highenergy range. On the other hand, GAF-EBT2 has been shown to be capable of measuring the second HVL, because it shows only slight energy dependency errors in comparison with GAF$\mathrm{R}$ and other radiochromic films [8,11-15]. For these reasons, in HVL measurements of devices operating in the high-energy range (X-ray $\mathrm{CT}$, radiotherapy machines, and so on), it is suggested that GAF-EBT2 offers higher measurement precision than GAF-R. Furthermore, GAF-EBT2 has several other advantages: the film size is ideally suited for this study, the dose range is $0.01-10 \mathrm{~Gy}$, and the density change is stabilized rapidly after exposure so that films can be processed in real time. Based on these results, the difference ratios in the effective energies obtained by the GAF-EBT2 and standard methods with an IC dosimeter were less than about $5 \%$ in a previous experiment [6].

However, as a disadvantage of GAF-EBT2, subtraction processing of the scanning image before and after exposure is necessary in order to maintain the precision of the HVL measurement, because of a serious uniformity error arises when GAF-EBT2 is subjected to a transmission mode scan. Therefore, the HVL measurement method using GAF-EBT2 is difficult because it requires a special process; subtraction processing. In addition, a transmission mode scan is problematic. A film scanner of A4 size or a flat-bed scanner with the transmission mode unit (a film adapter unit) is necessary. Although a more portable mobile scanner would be preferable for monitoring the effective energy of the X-ray generators constantly, mobile scanners do not typically offer a transmission mode. It was suggested that by scanning the GAFEBT2 using reflection mode, a low absorbed dose range, such as less than $100 \mathrm{mGy}$, could be measured without the nonuniformity error associated with transmission mode [16,17]. Therefore, all the scans of this study were performed in reflection mode.

The purpose of this study was to develop a method to evaluate the HVL simply, constantly, and quickly and at any time or place. Therefore, to measure the HVL with a mobile scanner, HVL measurement using GAF-EBT2 as the radiochromic film and a SSAl filter was performed in reflection scanning mode. The results of this GAF-EBT2 method using both a mobile scanner and a flat-bed scanner were compared with those of the standard method using an IC dosimeter in order to evaluate its applicability.

\section{Material and methods}

\section{GAFCHROMIC EBT2 Dosimetry Film (GAF- EBT2)}

GAF-EBT2 is rectangular in shape, measuring $8 " \times 10^{\prime \prime}$ $(20.5 \mathrm{~cm} \times 25.5 \mathrm{~cm})$; its specified thickness is $285 \mu \mathrm{m}$, and it is yellow in color. The scan mode recommended by the manufacturer for image acquisition is transmission type. However, this study was performed using reflection mode as this improves the precision of the measurement in the low absorbed dose range. When the active component is exposed to radiation, it changes to a dark-blue color. GAF-EBT2 is suitable for HVL measurement of X-rays in the diagnostic range, because it is recommended for dosimetry in the low dose range (0.01-10 Gy). In addition, its energy response character is minimal (within 10\%) from $\mathrm{keV}$ to the $6 \mathrm{MV}$ range $[8,14]$. The analysis time is shortened by scanning immediately after exposure, because density changes stabilize rapidly after exposure [18]. Disadvantages are that some non-uniformity errors of a few percent are caused by the coating in the directions of the short axis and long axis [7]. The GAF-EBT2 was kept at room temperature, $20-25^{\circ} \mathrm{C}$, in a shaded bag.

\section{Characteristic of flat-bed scanner and mobile scanner}

In this study, a flat-bed scanner (Epson ES-2200, Seiko Epson Co., Nagano) (Figure 1) and a mobile scanner (Yashica S40, Exemode Inc., Tokyo) (Figure 2) were used for digital image acquisition of GAF-EBT2. The read sensors of the flat-bed scanner and a mobile scanner are a charge coupled device (CCD) and a contact image sensor (CIS), respectively [19,20]. The flat-bed scanner is able to scan a film in transmission mode by attaching a film adapter unit (EPSON ESA4FLU). For the mobile scanner, transmission mode scanning is impossible because no such adapter exists. However, the mobile scanner has characteristics of low cost and the portability compared with a flat-bed scanner. To monitor the effective energy of $\mathrm{X}$ ray generators constantly, a mobile scanner is ideal.

The calibration curves of a mobile scanner and the flat-bed scanner were provided to evaluate the straightness of the calibration curve for the HVL measurement using GAF-EBT2. To make the calibration curve of absorbed dose versus Net Pixel Value (NPV), 10 regions (50 pixels in diameter) on the GAF-EBT2 sheet were exposed in the range between approximately $0-120 \mathrm{mGy}$ in increments of about $12 \mathrm{mGy}$. The exposure parameters were $120 \mathrm{kV}, 250 \mathrm{~mA}, 0.4 \mathrm{sec}$, and 2.0 $\mathrm{mm}$ additional $\mathrm{Al}$ filter. Exposure dose was measured with an IC dosimeter (Radcal-1015, $6 \mathrm{~cm}^{3}$ ) to monitor the X-ray output of the X-ray generator. The GAF-EBT2 and an IC dosimeter were placed at a distance of $1000 \mathrm{~mm}$ from the X-ray tube. To reduce the influence of radiation scattered from the floor, the dosimeter was placed $500 \mathrm{~mm}$ from the floor. The exposed GAF-EBT2 is shown in Figure 3. 


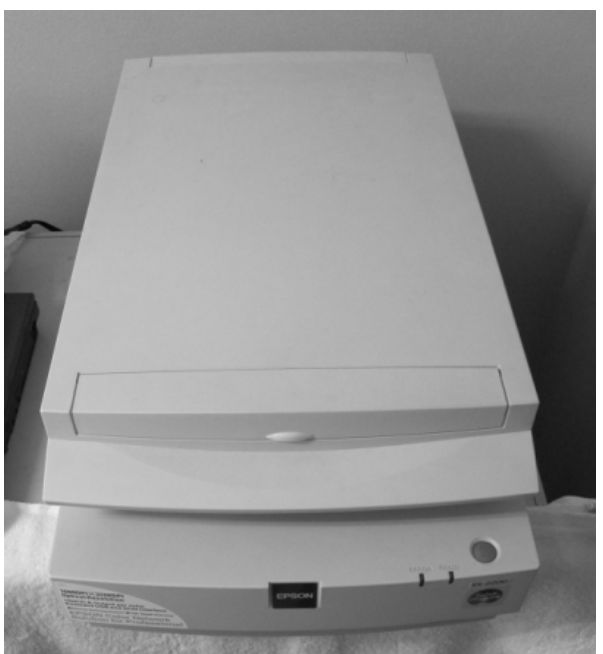

Figure 1. Flat-bed scanner (EPSON ES-2200, Seiko Epson Co., Nagano). The read sensors is the charge coupled device (CCD) system. The flat-bed scanner is able to scan a film of transmission mode by attaching a film adapter unit (EPSON ESA4FLU).

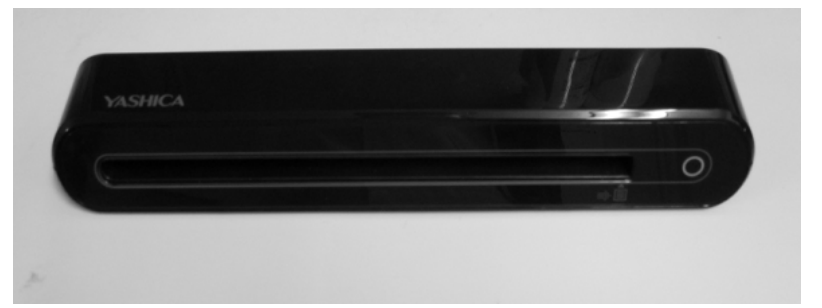

Figure 2. Mobile scanner (YASHICA S40, EXEMODE, INC., Tokyo). The read sensors is the contact image sensor (CIS) system. Transmission mode scan is impossible, because there is not a film adapter unit to a mobile scanner.

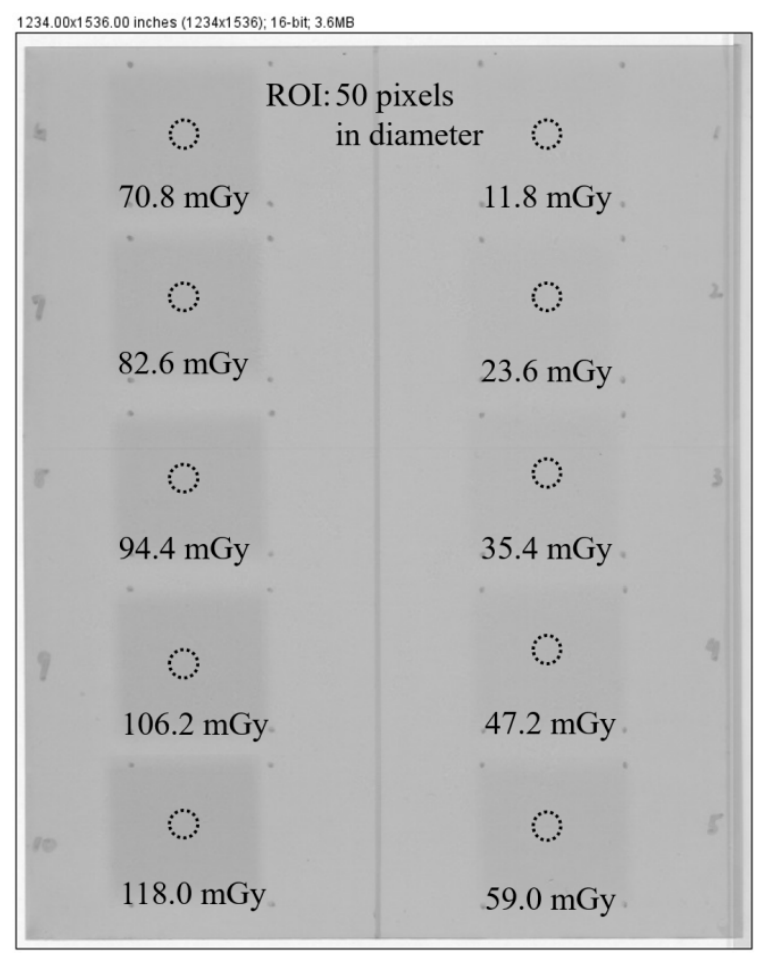

Figure 3. Scanned image of the GAF-EBT2 after exposure for calibration curve. ROIs on the GAF-EBT2 are shown as white line circle, sized diameter 50 pixels.
The GAF-EBT2 was scanned using a flat-bed scanner and a mobile scanner, in RGB (48 bit) mode, 150 dpi, with the protection of a film of liquid crystal (LCD-150, Sanwa Supply Inc., Okayama, Japan) for removal of moire artifacts. To scan GAF-EBT2 with reflection mode setting, white paper of uniform density was attached to back of the GAF-EBT2. Similarly, white paper of uniformity density was attached to GAF-EBT2 for scanning using a mobile scanner in RGB (48 bit) mode and $150 \mathrm{dpi}$. The scan direction was performed parallel to the long-axis direction of the GAF-EBT2 (the axis of the light source was a vertical direction to the long-axis direction).

The image data of the GAF-EBT2 were divided into R, G, and $\mathrm{B}$ modes (each 16 bit), and the $\mathrm{R}$ mode was used. It was converted to a gray scale and was analyzed using Image $\mathrm{J}$ version $1.48 \mathrm{v}$ image analysis software (National Institute of Health, Maryland). The region of interesting (ROI) was a diameter of 50 pixels in the center of each absorbed dose areas on GAF-EBT2 sheet, and it was averaged. The quantity of density increase was set as the NPV. Then, the calibration curves using a flat-bed scanner and a mobile scanner were provided using the NPV and absorbed dose.

\section{HVL measurement of the GAF-EBT2}

A SSAl filter of more than $99.8 \%$ purity was designed. The SSAl filter has a rectangular form, with dimensions of $20 \mathrm{~mm} \mathrm{x}$ $100 \mathrm{~mm}$, and its thickness increases from $1 \mathrm{~mm}$ to $25 \mathrm{~mm}$ (the specific thicknesses of the steps are 1, 2, 3, 5, 7.5, 10, 12.5, 15, 20 , and $25 \mathrm{~mm}$ ). The inherent filtration of the X-ray tube was $1.1 \mathrm{~mm} \mathrm{Al}$ and no further filtration was added.

HVL measurements were performed by setting the exposure parameters of the X-ray generator (DRX-2924HD, Toshiba Medical, Tochigi) to $120 \mathrm{kV}, 250 \mathrm{~mA}, 4.0 \mathrm{~s}(0.4 \mathrm{~s} \times 10) ; 100$ $\mathrm{kV}, 250 \mathrm{~mA}, 5.0 \mathrm{~s}(0.5 \mathrm{~s} \times 10)$; and $80 \mathrm{kV}, 320 \mathrm{~mA}, 5.0 \mathrm{~s}$ $(0.5 \mathrm{~s} \times 10)$. The anode-cathode direction of the X-ray tube was set parallel to the long axis of the GAF-EBT2 to minimize the impact of the X-ray heel effect on each $\mathrm{Al}$ filter thickness.

The thickness of the aluminum was varied in the long-axis direction of the GAF-EBT2. A central $100 \mathrm{~mm}$ band of the GAF-EBT2 was set as the exposure area, and lead masking plates (thickness $2 \mathrm{~mm}$ ) were placed on either side of the exposure area to create unexposed areas. The SSAl filter was placed in the center of the exposure area. A full-exposure area, an unexposed area, and a SSAl filter area were thus created on the GAF-EBT2. The full-exposure area was used to compensate for a non-uniformity error caused by the active layer of the GAF-EBT2, while the unexposed area was used to compensate for a non-uniformity error caused by the protective layer of the polyester. 


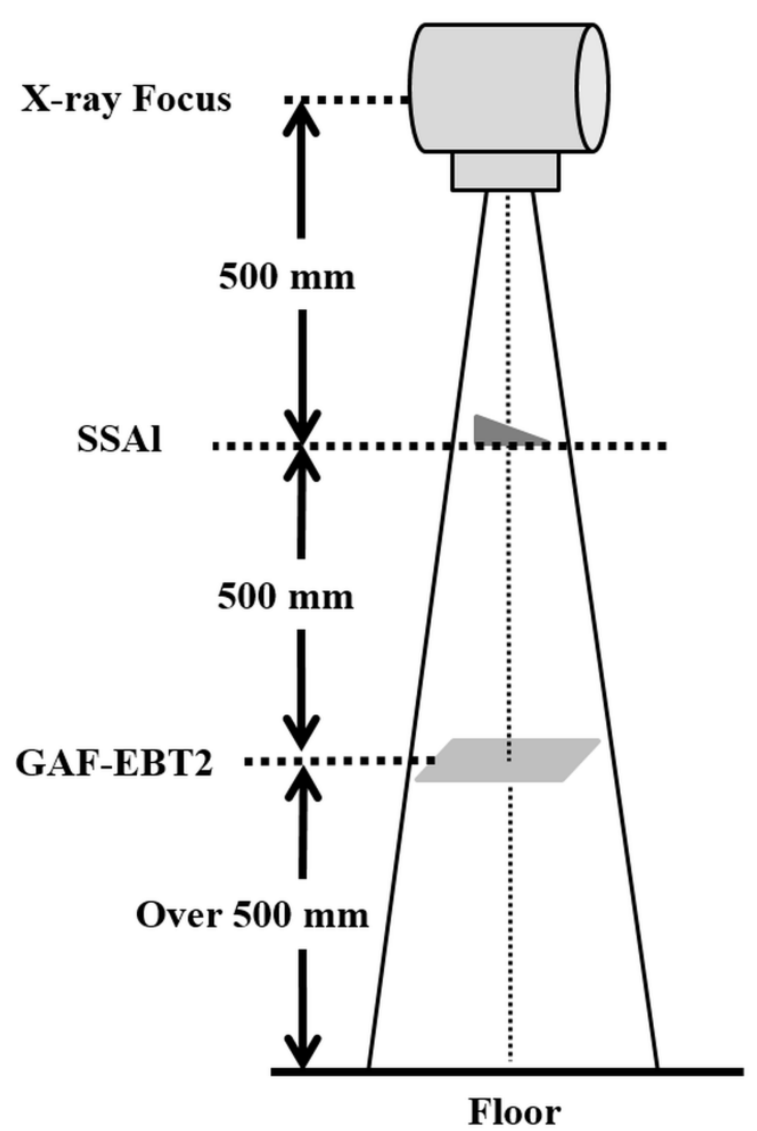

Figure 4. Geometric arrangement of the exposure method for HVL measurement. The distances between the X-ray focus and the SSAl filter and between the SSAl filter and the GAF-EBT2 were each $500 \mathrm{~mm}$. The distance from the floor to the GAF-EBT2 was over $500 \mathrm{~mm}$.

The geometric arrangement of the experimental set-up of the exposure method for measuring the HVL is shown in Figure 4. The distance from the X-ray focus to the SSAl filter was set at $500 \mathrm{~mm}$ to reduce the influence of diagonally incident X-rays. The distance from the SSAl filter to the GAF-EBT2 was also set at $500 \mathrm{~mm}$ to reduce the influence of scattered radiation from the SSAl filter. To avoid back-scattered radiation from the floor, the distance between the floor and the GAF-EBT2 was set at more than $500 \mathrm{~mm}$.

The scan method for the GAF-EBT2 film was performed in a similar manner as the method in above section. The GAFEBT2 was scanned using a flat-bed scanner and a mobile scanner in RGB (48 bit) mode, $150 \mathrm{dpi}$, with the protection of a film of liquid crystal. The GAF-EBT2 was scanned by setting the scanner to reflection mode. The scan direction was performed parallel to the long-axis direction of the GAF-EBT2.

The respective image data of the GAF-EBT2, generated by the flat-bed scanner and mobile scanner, were divided into R, $\mathrm{G}$, and B modes (each 16 bit), and the R mode was used. This was converted to a gray scale, inverted to black and white, and analyzed using Image J. In this study, HVLs for X-ray tube voltages of $120 \mathrm{kV}, 100 \mathrm{kV}$, and $80 \mathrm{kV}$ were measured using
1-15 mm, 1-10 mm, and 1-7.5 mm thicknesses of the SSAl filter, respectively.

The ROI was set at each thickness of the SSAl filter and was sized 900 pixels $(152.4 \mathrm{~mm}) \times 20$ pixels $(3.4 \mathrm{~mm})$. The ROI on the GAF-EBT2 included a sequence of an unexposed area, a full-exposure area, the SSAl filter area, a full-exposure area, and an unexposed area. In terms of density, the densities of an exposure area and an unexposed area were set at $100 \%$ and $0 \%$, respectively, and the density ratio of the SSAl filter area could then be calculated. The attenuation curve for HVL measurement was obtained using the density ratio of each SSAl filter thickness. The HVL was then calculated using the attenuation curve. To observe the energy dependence of the GAF-EBT2, the second HVL was calculated throughout. In addition, the effective energy was obtained from the HVL. The conversion from HVL to effective energy $(\mathrm{HVL} \Rightarrow$ linear attenuation coefficient $\Rightarrow$ mass attenuation coefficient $\Rightarrow$ effective energy) was calculated using the basic data of Seltzer and Hubbell at the National Institute of Standards and Technology [21].

\section{HVL measurement by the standard method using an IC dosimeter}

HVL measurement using an IC dosimeter (Radcal-1015, $6 \mathrm{~cm}^{3}$ ) was performed for comparison with the method using GAF-EBT2 and a SSAl filter. The exposure parameters were $120 \mathrm{kV}, 200 \mathrm{~mA}, 0.05 \mathrm{~s} ; 100 \mathrm{kV}, 200 \mathrm{~mA}, 0.05 \mathrm{~s}$; and $80 \mathrm{kV}$, $200 \mathrm{~mA}, 0.1 \mathrm{~s}$. The thicknesses of the Al filter were 1, 2, 3, 5, $7.5,10,12.5$, and $15 \mathrm{~mm}$. A normal geometric arrangement of the exposure method for measuring the HVL based on Japanese Industrial Standards was adopted to minimize the influence of scattered radiation. Exposure and dose measurements were performed three times for each $\mathrm{Al}$ thickness, and averaged dose values were used. Observed dose values $(\mathrm{mR})$ of the IC with correction for temperature and atmospheric pressure were used, because the attenuation curve was calculated using a relative dose value. Temperature correction was performed automatically by the IC. The HVLs were then calculated using the attenuation curves of the observed dose values at $120 \mathrm{kV}, 100 \mathrm{kV}$, and $80 \mathrm{kV}$. In addition, the second HVLs were calculated. The effective energies were then calculated using the basic data of Seltzer and Hubbell [21].

For comparison of the respective data of the two scanners, as well as the standard method using the IC dosimeter, attenuation curves were generated. The results of the method using the mobile scanner in reflection mode were compared with the method using the flat-bed scanner in reflection mode, and the results of reflection mode using the two kinds of scanners were compared with those of the standard method using IC dosimetry, in order to evaluate its applicability. 


\section{Results}

\section{Calibration curves of the GAF-EBT2 using a flat-} bed scanner and a mobile scanner

The calibration curves of the GAF-EBT2 using a flat-bed scanner and a mobile scanner are shown in Figure 5. The coefficient of determination $\left(\mathrm{r}^{2}\right)$ for the straight-line approximation of the calibration curve using both the flat-bed scanner and a mobile scanner were higher than 0.9 (until about $120 \mathrm{mGy}$ ). As a result, the HVL can be evaluated using only the ratio of the density distribution in the GAF-EBT2.

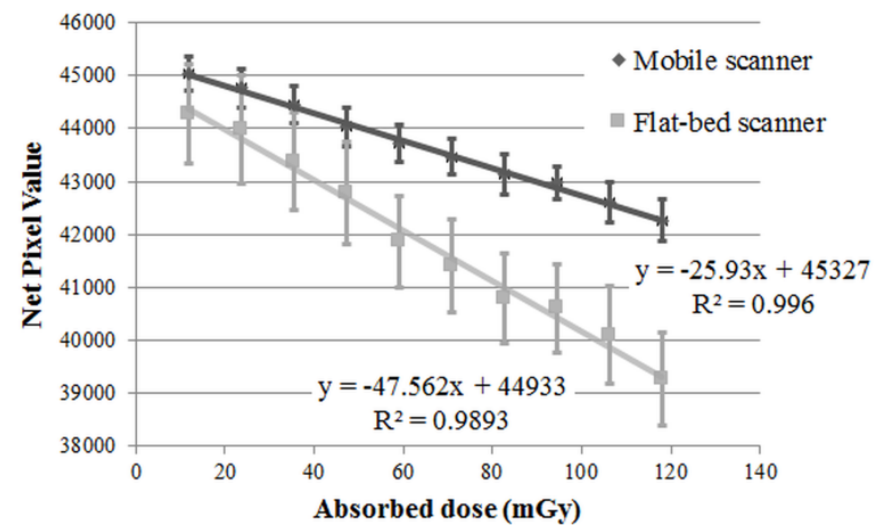

Figure 5. Calibration curves of the GAF-EBT2 using a flatbed scanner and a mobile scanner.

\section{Profile curves of the GAF-EBT2 using a flat-bed scanner and a mobile scanner}

Figure 6 shows density profile curves of the ROI $(120 \mathrm{kV}$ and Al thickness $5 \mathrm{~mm}$ ) using the flat-bed scanner and the mobile scanner. The profile curve of the mobile scanner showed a lower density compared to the flat-bed scanner and it showed a wider range of the density. Both the flat-bed scanner and the mobile scanner provided flat density profile curves.

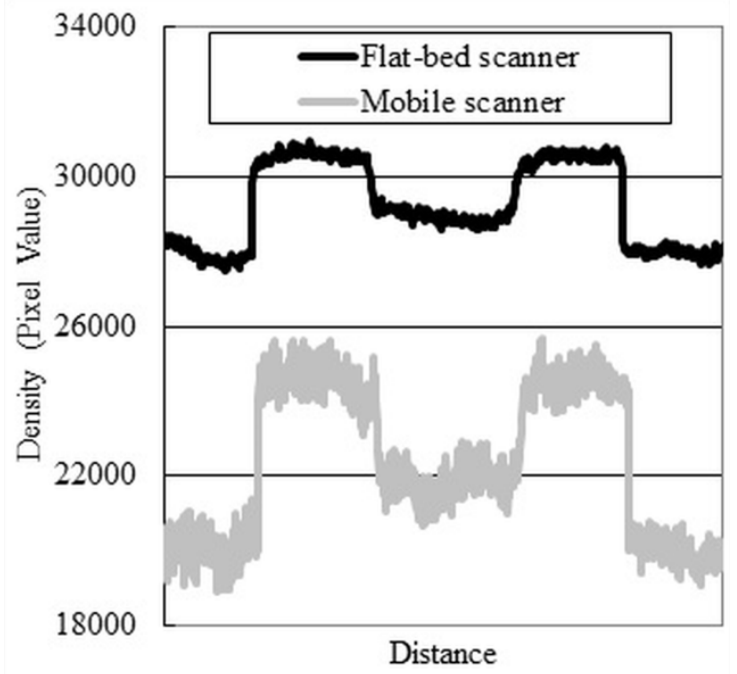

Figure 6. Density profile curves of the ROI $(120 \mathrm{kV}$ and $\mathrm{Al}$ thickness $5 \mathrm{~mm}$ ) using a flat-bed scanner and a mobile scanner.

\section{Attenuation curves using GAF-EBT2 (flat-bed scanner or mobile scanner) and IC dosimeter}

Figure 7 shows the attenuation curves for the methods using the flat-bed scanner and the mobile scanner, respectively. Figures $7 \mathbf{a}$ ), b), and c) correspond to X-ray tube voltages of $120 \mathrm{kV}, 100 \mathrm{kV}$, and $80 \mathrm{kV}$, respectively. The attenuation curves at $120 \mathrm{kV}, 100 \mathrm{kV}$, and $80 \mathrm{kV}$ using GAF-EBT2 in transmission mode with subtraction are from a previous study [6]. The characteristics of each attenuation curve were most obvious at $120 \mathrm{kV}$. The attenuation curves using the GAFEBT2 were similar to those obtained by the standard method using an IC dosimeter. These attenuation curves thus showed a similar curve from between attenuation ratios of 1 and 0.5 .
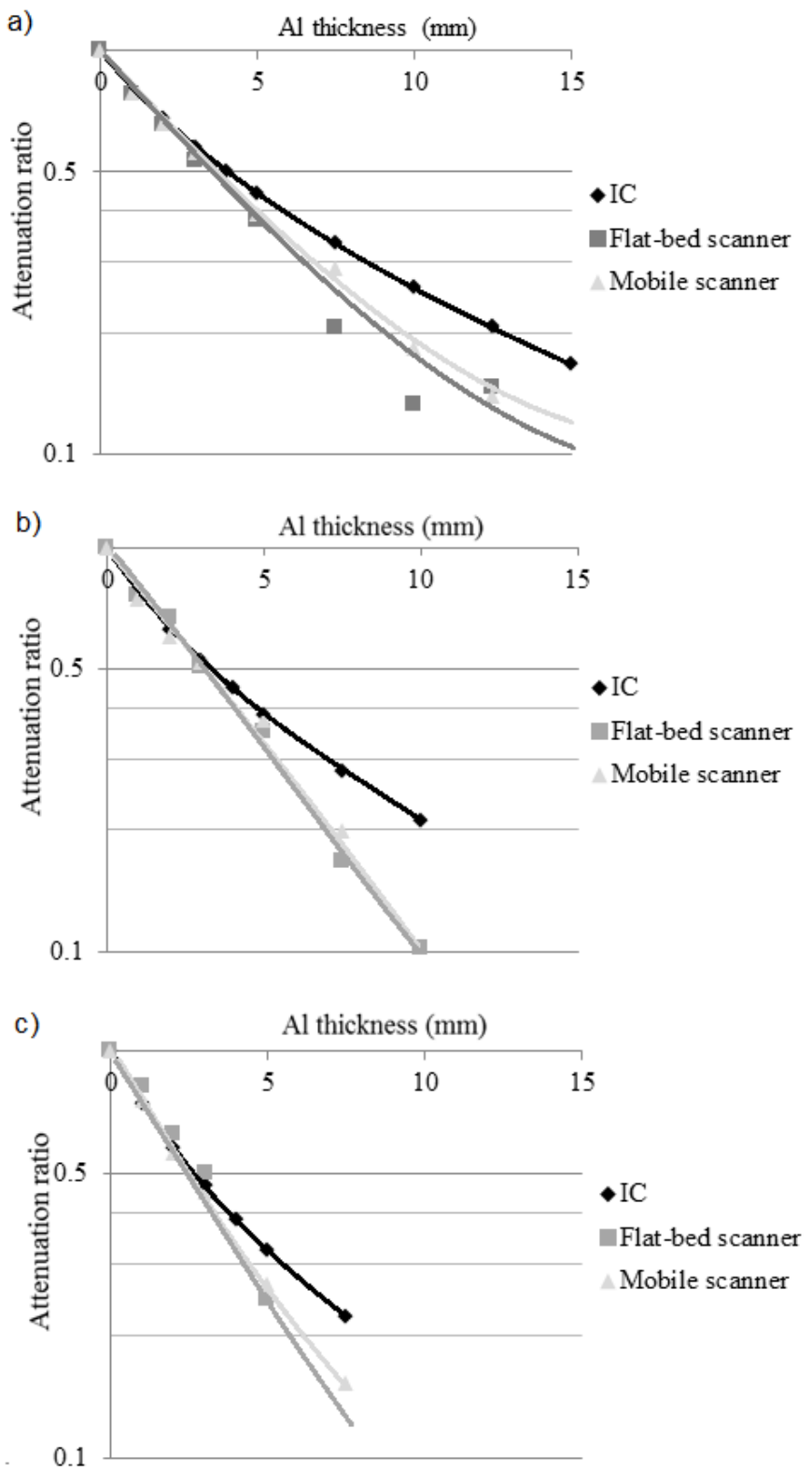

Figure 7. Attenuation curves in the method using a flat-bed scanner and a mobile scanner with GAF-EBT2, and the standard method with an IC dosimeter: a) $120 \mathrm{kV}$, b) $100 \mathrm{kV}$, c) $80 \mathrm{kV}$. GAF-EBT2: GAFCHROMIC EBT2; IC: ionization chamber. 


\section{Comparison of the collected data on HVLs and effective energies using GAF-EBT2 (flat-bed scanner or mobile scanner) and an IC dosimeter}

In the standard method using an IC dosimeter, the HVLs (second HVLs) at $120 \mathrm{kV}, 100 \mathrm{kV}$, and $80 \mathrm{kV}$ were $4.03 \mathrm{~mm}$ $(10.35 \mathrm{~mm}), 3.30 \mathrm{~mm}(8.46 \mathrm{~mm})$, and $2.68 \mathrm{~mm}(6.67 \mathrm{~mm})$, respectively, and the effective energies at these voltages were $38.7 \mathrm{keV}, 35.3 \mathrm{keV}$, and $32.2 \mathrm{keV}$, respectively. Table 1 shows the HVLs, the effective energy and the second HVLs at 120 $\mathrm{kV}, 100 \mathrm{kV}$, and $80 \mathrm{kV}$ for each method. The differences in ratios of each result compared to IC are shown next to the results as percentages. The difference ratios of the effective energies by each methods using GAF-EBT2 were less than $5 \%$ at each tube voltage.

Table 1. HVLs, the effective energy and the second HVLs at $120 \mathrm{kV}, 100 \mathrm{kV}$, and $80 \mathrm{kV}$ using a flat-bed scanner and a mobile scanner with GAF-EBT2, and the standard method using IC dosimeter. HVL: half-value layer; GAF-EBT2: GAFCHROMIC EBT2; IC: ionization chamber.

\begin{tabular}{|c|c|c|c|c|c|c|}
\hline \multirow{2}{*}{\multicolumn{2}{|c|}{$\begin{array}{c}\text { Dosimeter } \\
\text { Scanner }\end{array}$}} & \multirow{2}{*}{$\begin{array}{c}\text { IC } \\
-\end{array}$} & \multicolumn{4}{|c|}{ GAF-EBT2 } \\
\hline & & & \multicolumn{2}{|c|}{ Flat-bed scanner } & \multicolumn{2}{|c|}{ Mobile scanner } \\
\hline \multirow{3}{*}{$120 \mathrm{kV}$} & HVL [mm] & 4.03 & 3.67 & $(-9.0 \%)$ & 3.68 & $(-8.8 \%)$ \\
\hline & $\mathrm{EE}[\mathrm{keV}]$ & 38.7 & 37.0 & $(-4.3 \%)$ & 37.1 & $(-4.2 \%)$ \\
\hline & $2^{\text {nd }}$ HVL $[\mathrm{mm}]$ & 10.35 & 7.34 & $(-29.1 \%)$ & 8.07 & $(-22.0 \%)$ \\
\hline \multirow{3}{*}{$100 \mathrm{kV}$} & HVL [mm] & 3.30 & 3.12 & $(-5.3 \%)$ & 3.30 & $(0.0 \%)$ \\
\hline & $\mathrm{EE}[\mathrm{keV}]$ & 35.3 & 34.4 & $(-2.4 \%)$ & 35.3 & $(0.0 \%)$ \\
\hline & $2^{\text {nd }} H V L[\mathrm{~mm}]$ & 8.46 & 6.13 & $(-27.5 \%)$ & 6.32 & $(-25.3 \%)$ \\
\hline \multirow{3}{*}{$80 \mathrm{kV}$} & HVL [mm] & 2.68 & 2.88 & $(7.2 \%)$ & 2.51 & $(-6.3 \%)$ \\
\hline & $\mathrm{EE}[\mathrm{keV}]$ & 32.2 & 33.2 & $(3.1 \%)$ & 31.3 & $(-2.8 \%)$ \\
\hline & $2^{\text {nd }}$ HVL $[\mathrm{mm}]$ & 4.03 & 5.06 & $(-24.2 \%)$ & 5.32 & $(-20.3 \%)$ \\
\hline
\end{tabular}

\section{Discussion}

\section{Reflection mode using white paper of uniform density}

Radiochromic film can be measured in two-dimensions in the same way as a film dosimeter. Based on this characteristic, it was possible to evaluate the HVL with a method using radiochromic film and a SSAl filter. In a previous study, a method for obtaining precise data that compensated for the non-uniformity errors of the active layer and the protective layer of the radiochromic film was established [5,6]. In this compensation method, the full-exposure area, the unexposed area, and the SSAl filter area are examined at each filter thickness [5], and in this way the HVL measurement using GAF-R with reflection type can be performed with high precision. However, the transmission scan data for GAF-EBT2 showed a uniformity error caused by the transmission mode scan of the flat-bed scanner. In other words, the results were affected by the distance between the GAF-EBT2 and the light source of the scanner. The maximum scan size $(21.6 \mathrm{~cm} \times 29.7$ $\mathrm{cm}$ ) of the scanner used in this study was just larger than the size of the GAF-EBT2 $(20.5 \mathrm{~cm} \times 25.5 \mathrm{~cm})$. It is suggested that the light on either side became slightly weak, even though the light source of the scanner was in a parallel orientation. This uniformity error could be almost eliminated by using reflection mode (flat-bed scanner) instead of transmission mode. We consider that the light source of the scanner in reflection mode is more uniform than that in transmission mode. The difference ratios in the effective energies using a flat-bed scanner (reflection mode) compared with the standard methods were less than 5\%. Japanese Industrial Standards require that the measurement precision be less than 5\%. Therefore, it is suggested that the method using a flat-bed scanner (reflection mode) with GAF-EBT2 may be of practical use for QA and QC in all institutions. In addition, this processing is very effective when scanning before exposure is impossible. However, GAF-EBT2 with reflection mode showed erroneously low values at the thicker end of the $\mathrm{Al}$ filter in comparison with transmission mode (with subtraction). To explain this, we consider that the route of the light in the radiochromic film is doubled by using reflection rather than transmission scanning. Therefore, although the method using GAF-EBT2 with subtraction processing was able to measure second HVL, the second HVL measurement in reflection mode has to be treated with caution.

\section{Measurement precision of the flat-bed scanner and mobile scanner}

The HVL measurement precision is influenced by the kind of the scanner as well as the kind of radiochromic film. In both the flat-bed scanner and a mobile scanner used in this study, there were several differences in the method and mechanism of image acquisition. Firstly, the mobile scanner only offers reflection mode. Therefore, reflection mode was used in order to scan GAF-EBT2 using a mobile scanner. Although differences in density dynamic range and quantum noise graininess were produced, the profile curve was as flat as with a flat-bed scanner.

Secondly, the light sources of the flat-bed scanner and mobile scanner are a high-intensity xenon lamp and a lightemitting diode (LED), respectively. Although there is an overall density difference on the acquired image, these errors were eliminated by compensation processing in which the density of a fully-exposed area and an unexposed area were set at $100 \%$ and $0 \%$, respectively. In addition, the LED light source used in the mobile scanner is advantageous in that scanning is performed immediately without the need for a warm-up to stabilize the light source.

Thirdly, the read sensors of the flat-bed scanner and mobile scanner are CCD and CIS systems, respectively. In this study, we considered that there was little influence of the read sensor type, because the GAF-EBT2 was closely attached to the surface of scanner with the protection of a film of liquid crystal.

Fourthly, we must consider movement of the radiochromic film at the time of scaning. The flatbed scanner had high reproducibility, because the GAF-EBT2 was in a fixed position 
for scanning. Conversely, in the mobile scanner, the image had low reproducibility because the GAF-EBT2 moved during each. However, it is considered that plasticity is not important because the image obtained by reflection mode using the mobile scanner does not require subtraction processing.

In this way, although the method and mechanism of image acquisition are different, the measurement precision of the mobile scanner (reflection mode) was nearly equal to that from the flat-bed scanner (reflection mode). It is considered that the method using a mobile scanner has sufficient precision for constant monitoring because the mobile scanner was able to measure the effective energy with a difference of less than $5 \%$ compared with the standard method.

\section{Conclusions}

Changing the scan method from transmission mode to reflection mode using GAF-EBT2 is applicable for effective energy measurement, because the difference ratios in the effective energies compared with the standard method were less than about 5\%. In addition, this processing was very effective when scanning before the exposure was impossible. Based on this result, the mobile scanner proved capable of scanning GAF-EBT2 in reflection mode. The method using a mobile scanner with GAF-EBT2 described herein has portability advantages in HVL measurements for the evaluation of effective energy compared with the method using a flat-bed scanner. To constantly monitor the effective energy of X-ray generators, a mobile scanner is ideal. In addition, although the method and mechanism of the image acquisition were different, the measurement precision of the mobile scanner was nearly equal to that of the flat-bed scanner. The method using the mobile scanner offers sufficient precision for constant monitoring because the mobile scanner was able to measure the effective energy with a precision error of less than $5 \%$. In the light of these findings, it is considered that the method using a mobile scanner with GAF-EBT2 offers a simple means of determining HVL for QA and QC consistently and quickly and at any time or place, without the need for an IC dosimeter.

\section{Acknowledgements}

This study was supported by JSPS KAKENHI Grant Number $16 \mathrm{~K} 21553$.

\section{References}

[1] Shepard SJ, Lin PP, Boone JM, et al. Quality control in diagnostic radiology. Madison, WI: Medical Physics Publishing, AAPM Report No 74; 2002.

[2] Butson MJ, Yu PKN, Cheung T, et al. Radiochromic film for medical radiation dosimetry. Mat Sci EngR, 2003;41(3): 61-120.

[3] Cheung T, Butson MJ, Yu PK. Reflection spectrometry analysis of irradiated GAFCHROMIC XR type R radiochromic films. Appl Radiat Isot, 2005;63(1):127-129.

[4] Gotanda T, Katsuda T, Gotanda R, et al. Evaluation of effective energy for QA and QC: measurement of half-value layer using radiochromic film density. Australas Phys Eng Sci Med. 2009;32(1),26-29.

[5] Gotanda T, Katsuda T, Gotanda R, et al. Half-value layer measurement: simple process method using radiochromic film. Australas Phys Eng Sci Med. 2009;32(3):150-158.

[6] Gotanda T, Katsuda T, Gotanda R, et al. Evaluation of effective energy using radiochromic film and a step-shaped aluminum filter. Australas Phys Eng Sci Med. 2011;34(2):213-222.

[7] International Specialty Products [Internet]. [cited 2016 October 18]. Available from: http://www.veritastk.co.jp/attached/2062/GAFCHROMICEBT2TechnicalBrief-Rev1.pdf

[8] Sutherland JG, Rogers DW. Monte Carlo calculated absorbed-dose energy dependence of EBT and EBT2 film. Med Phys. 2010;37(3):1110-1116.

[9] Dini SA, Koona RA, Ashburn JR, et al. Dosimetric evaluation of GAFCHROMIC XR type T and XR type R films. J Appl Clin Med Phys. 2005;6(1):114-134.

[10] Butson MJ, Cheung T, Yu PK. XR type-R radiochromic film x-ray energy response. Phys Med Biol. 2005;50(15):N195-N199.

[11] Fletcher CL, Mills JA. An assessment of GafChromic film for measuring $50 \mathrm{kV}$ and $100 \mathrm{kV}$ percentage depth dose curves. Phys Med Biol. 2008;53(11):N209-N218.

[12] Butson MJ, Cheung T, Yu PK. Weak energy dependence of EBT gafchromic film dose response in the $50 \mathrm{kVp}-10 \mathrm{MVp}$ X-ray range. Appl Radiat Isot. 2006;64(1):60-62.

[13] Tomic N, Devic S, DeBlois F, et al. Reference radiochromic film dosimetry in kilovoltage photon beams during CBCT image acquisition. Med Phys. 2010;37(3):1083-1092.

[14] Butson MJ, Yu PK, Cheung T, et al. Energy response of the new EBT2 radiochromic film to x-ray radiation. Radiat Meas. 2010;45(7):836-839.

[15] Rink A, VitkinIA, Jaffray DA. Energy dependence ( $75 \mathrm{kVp}$ to $18 \mathrm{MV}$ ) of radiochromic films assessed using a real-time optical dosimeter. Med Phys. 2007;34(2):458-463. 
[16] Gotanda T, Katsuda T, Akagawa T, et al. Evaluation of GAFCHROMIC EBT2 dosimetry for the low dose range using a flat-bed scanner with the reflection mode. Australas Phys Eng Sci Med. 2013;36(1):59-63.

[17] Butson E, Alnawaf H, Yu PK, et al. Scanner uniformity improvements for radiochromic film analysis with matt reflectance backing. Australas Phys Eng Sci Med. 2011;34(3):401-407.

[18] Cheung T, Butson MJ, Yu PK. Post-irradiation colouration of Gafchromic EBT radiochromic film. Phys Med Biol. 2005;50(20):N281-N285.

[19] EPSON [Internet]. [cited 2009 August 15]. Available from: http://www.epson.jp/support/manual/scanner/es2200.htm/

[20] exemode [Internet]. [cited 2010 November 15]. Available from: http://www.exemode.com/yashica/fs/s40.html/

[21] Seltzer SM, Hubbell JH. Tables and Graphs of Photon Mass Attenuation Coefficients and Mass Energy-Absorption Coefficients for Photon Energies $1 \mathrm{keV}$ to $20 \mathrm{MeV}$ for Elements $\mathrm{Z}=1$ to 92 and Some Dosimetric Materials. National Institute of Standards and Technology; 1995. 